\title{
An optical frequency comb for infrared spectrograph calibration
}

\author{
Gabriel G. Ycas ${ }^{a b}$, Franklyn Quinlan ${ }^{b}$, Steve Osterman ${ }^{c}$, Gillian Nave ${ }^{d}$, Scott A. Diddams ${ }^{b}$ \\ ${ }^{a}$ Department of Physics, University of Colorado, Boulder, Colorado, USA \\ ${ }^{b}$ National Institute of Standards and Technology, 325 Broadway, Boulder, CO, USA \\ ${ }^{c}$ University of Colorado, Center for Astrophysics and Space Astronomy, Boulder, CO, USA \\ ${ }^{d}$ National Institute of Standards and Technology, 100 Bureau Drive, Gaithersburg, MD, USA
}

\section{INTRODUCTION}

The search for extrasolar planets is an exciting new field of astronomy. Since detection of a planet orbiting the sun-like star $51 \mathrm{Peg},{ }^{1}$ the field of planet finding has pushed the limits of sensitivity and accuracy in astronomical photometry and spectroscopy. To date 455 exoplanets have been detected*, of which the radial velocity technique is responsible for nearly $80 \% .^{2}$ Radial velocity measurements are also an important complement to photometric missions such as Kepler and CoRoT, which survey vast numbers of stars simultaneously but which require follow up measurements for positive identification of planets.

The chief objective in the search for exoplanets is the identification of habitable Earth-like planets in close proximity to our solar system. Of the currently detected exoplanets, only a few are Earth-like, ${ }^{3}$ the vast majority being giants in close orbits. While it is possible that these planets are the most common type, it is likely that an inherent selection bias in planet finding techniques is the cause. Simply, large radial velocity shifts and high contrast occultations are the most detectable by radial velocity spectroscopy and photometry, and so we primarily observe planets capable of inducing them.

An Earth-like planet is a rocky planet existing in the habitable zone of a star-a range of orbital distances where the planet could support the existence of liquid water. Stellar lumninocity determines the location of the habitable zone and has significant implications for the detectability of Earth-like planets. The habitable zone of a G star is centered around 1 AU, while a cooler M star's zone is shifted inwards to 0.03-0.1 AU. ${ }^{4}$ The stellar radial velocity shift scales as $1 / \sqrt{r}, r$ being the planet's orbital radius, and accordingly the radial velocity signal for an Earth-like planet orbiting an $\mathrm{M}$ star will be 10 times greater than the same planet in the habitable zone of a G star, making cooler stars excellent targets for radial velocity measurements.

In addition to making attractive targets for radial velocity measurements, $\mathrm{M}$ stars are also our most numerous stellar neighbors. A survey of all stars within $10 \mathrm{pc}$ of our solar system indicates that 239 of the 354 such stars are $\mathrm{M}$ stars $^{\dagger}$. The stellar flux of an M star peaks at around $1000 \mathrm{~nm}$, making spectroscopy in the IR attractive. The $\mathrm{H}$ band atmospheric transmission window from 1400 to $1800 \mathrm{~nm}$ is an attractive spectral region for spectroscopy, with a relatively high density of spectral features, allowing for an intrinsic uncertainty of less than $5 \mathrm{~m} / \mathrm{s}^{5}$ Additionally, this region of the spectrum is a good match for fiber laser technology, as will be discussed in detail.

To achieve the accuracy required for meter per second and better radial velocity measurements, an accurate and highly stable calibration source is required. While thorium-argon lamps and iodine absorption cells can provide meter per second accuracy in the visible, ${ }^{6,7}$ in the $\mathrm{H}$ band both emission and absorption lines are sparse. Laser frequency combs provide an ideal calibration source for this spectral region.

Further author information: (Send correspondence to G. Ycas)

G.G.Y.: E-mail: gabriel.ycas@nist.gov, Telephone: 13034973177

F.Q.: E-mail: fquinlan@boulder.nist.gov

S.A.D: E-mail: sdiddams@boulder.nist.gov

S.O.: E-mail: steven.n.osterman@colorado.edu

${ }^{*}$ http://exoplanet.eu

${ }^{\dagger}$ See the RECONS census of nearby stars (http://www.recons.org) for an up-to-date listing.

Ground-based and Airborne Instrumentation for Astronomy III, edited by lan S. McLean,

Suzanne K. Ramsay, Hideki Takami, Proc. of SPIE Vol. 7735, 77352R - @ 2010

SPIE · CCC code: 0277-786X/10/\$18 - doi: 10.1117/12.857462

Proc. of SPIE Vol. 7735 77352R-1 


\section{LASER FREQUENCY COMBS}

Mode-locked laser frequency combs are well suited for the calibration of high resolution spectrographs. ${ }^{8-10}$ The frequency comb is an emission source, with a spectrum hundreds of nanometers broad and composed of thousands of spectral lines at regular intervals in the frequency or wavenumber domain.

Not only is the comb broad and densely populated, it also provides an absolute calibration, directly traceable to atomic frequency standards. The positions of the comb lines are determined completely by two parameters: the laser repetition rate $f_{\text {rep }}$, which sets the line spacing, and the carrier envelope offset frequency $f_{\text {ceo }}$. In terms of these two parameters, the locations of the comb lines are described by the simple equation

$$
f_{\mathrm{n}}=n \times f_{\text {rep }}+f_{\text {ceo }}
$$

where the mode index $n$ is a large integer on the order of $10^{6}$. Both $f_{\text {rep }}$ and $f_{\text {ceo }}$ can be measured and actively stabilized to an absolute frequency standard traceable to the SI second, allowing spectra recorded years apart or at different facilities to be directly compared.

\section{FREQUENCY COMB GENERATION AND STABILIZATION}

Our frequency comb is built upon a commercial passively mode-locked erbium doped fiber laser. ${ }^{11}$ The laser has two primary output ports, each providing $40 \mathrm{~mW}$, with a mode spacing of $250 \mathrm{MHz}$ and a spectral bandwidth of $\sim 70 \mathrm{~nm}$. One output port is used for the stabilization of the carrier envelope offset frequency, while the other is used to generate the calibration spectrum.

To stabilize the comb, $f_{\text {rep }}$ and $f_{\text {ceo }}$ are locked to global positioning system (GPS) disciplined local oscillator, providing accuracy of 1 part in $10^{11}$ and sufficient for a cm/s-level calibration. Direct detection and locking of the laser repetition rate is straightforward, as it is well within the bandwidth of inexpensive photodiodes. To

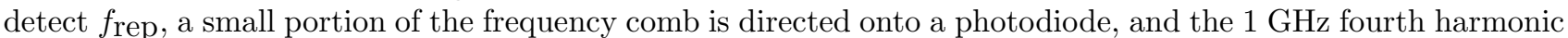
is amplified and mixed with a local oscillator signal provided by an RF frequency synthesizer; see Fig. 2 . The base band component of the mixed signal is fed into a loop filter, which provides an error signal. Feedback is applied to a piezo-mounted mirror inside the laser cavity, which stabilizes repetition rate.

Detection of the carrier envelope offset frequency is more involved. ${ }^{12}$ The carrier envelope offset is an absolute frequency shift, the residual frequency when equation Eq. 1 is extrapolated to $n=0$. Detection of $f_{\text {ceo }}$ is accomplished with the $\mathrm{f}-2 \mathrm{f}$ optical heterodyne technique. The frequency comb is amplified and broadened to span from 1000 to $2000 \mathrm{~nm}$, and a narrow band of the spectrum around $2000 \mathrm{~nm}$ is then frequency doubled in a periodically poled lithium niobate crystal. The resulting frequency doubled light is optically mixed with the $1000 \mathrm{~nm}$ portion of the spectrum on a photodiode, resulting in a signal at base band with frequency

$$
f_{0}=2\left(n \times f_{\text {rep }}+f_{\text {ceo }}\right)-\left(2 n \times f_{\text {rep }}+f_{\text {ceo }}\right),
$$

where the first term is the contribution of the frequency doubled $2000 \mathrm{~nm}$ light and the second is the $1000 \mathrm{~nm}$ light. The difference frequency $f_{0}$ is equal to $f_{\text {ceo }}$, and it is divided by 8 and phase locked locked to a synthesized 17.5 MHz reference frequency. The frequency synthesizers stabilizing $f_{\text {rep }}$ and $f_{\text {ceo }}$ are themselves referenced to a GPS disciplined rubidium oscillator with accuracy of 1 part in $10^{11}$, sufficient for sub-cm/s radial velocity calibration. 


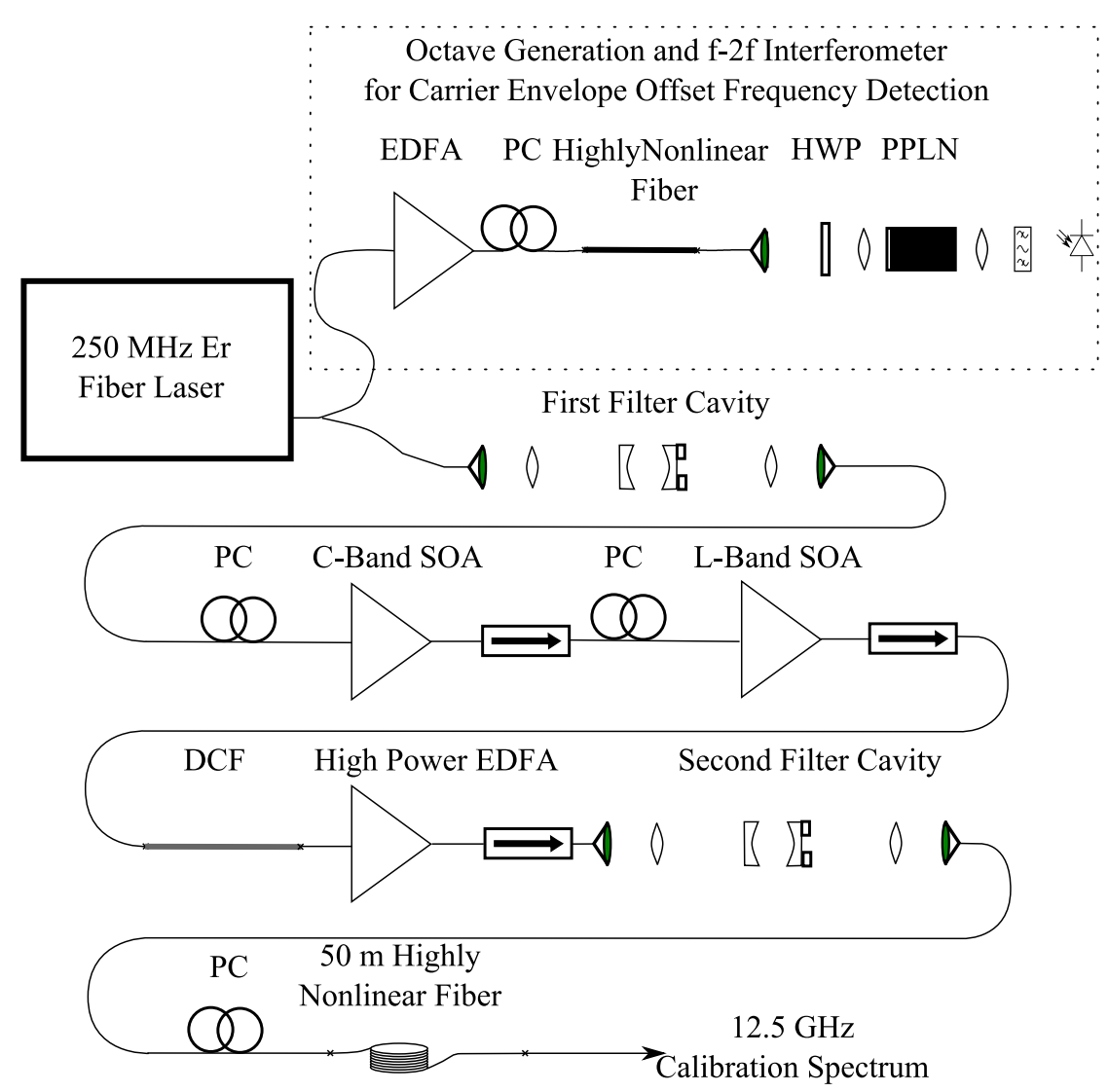

Figure 1: Schematic diagram of frequency comb optics. The upper block broadens the $250 \mathrm{MHz}$ frequency comb to span an octave, from 1000 to $2000 \mathrm{~nm}$, frequency doubles the $2000 \mathrm{~nm}$ portion of the spectrum, then photodetects the $\mathrm{f}-2 \mathrm{f}$ beat note (see text.) The optics in the lower block filter the $250 \mathrm{MHz}$ frequency comb by a factor of 50 to $12.5 \mathrm{GHz}$, then amplify, clean up, and broaden the spectrum for astronomical spectrograph calibration. EDFA: erbium doped fiber amplifier; PC: polarization control; HWP: half wave plate; SOA: semiconductor optical amplifier; DCF: dispersion compensating fiber; PPLN: periodically poled lithium niobate. Unless otherwise specified, optical fiber is SMF-28e. 


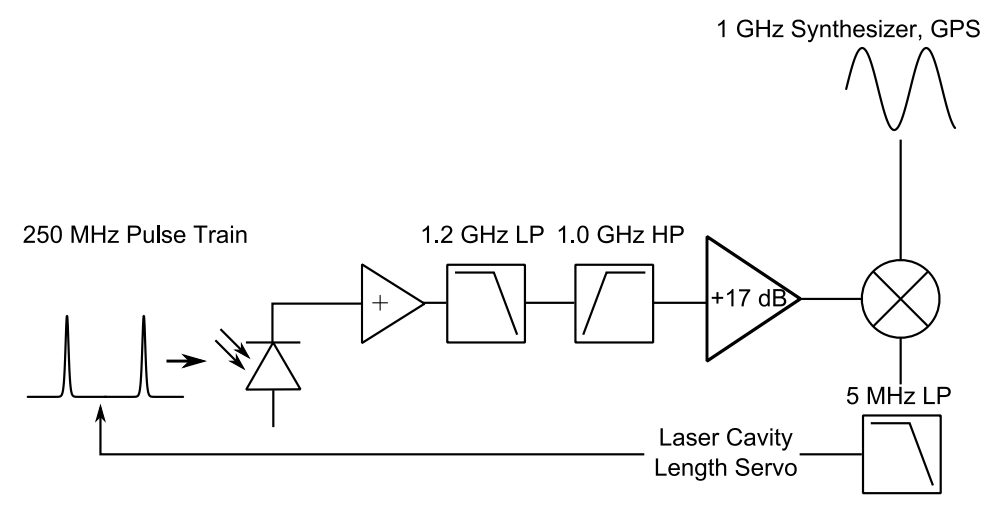

(a)

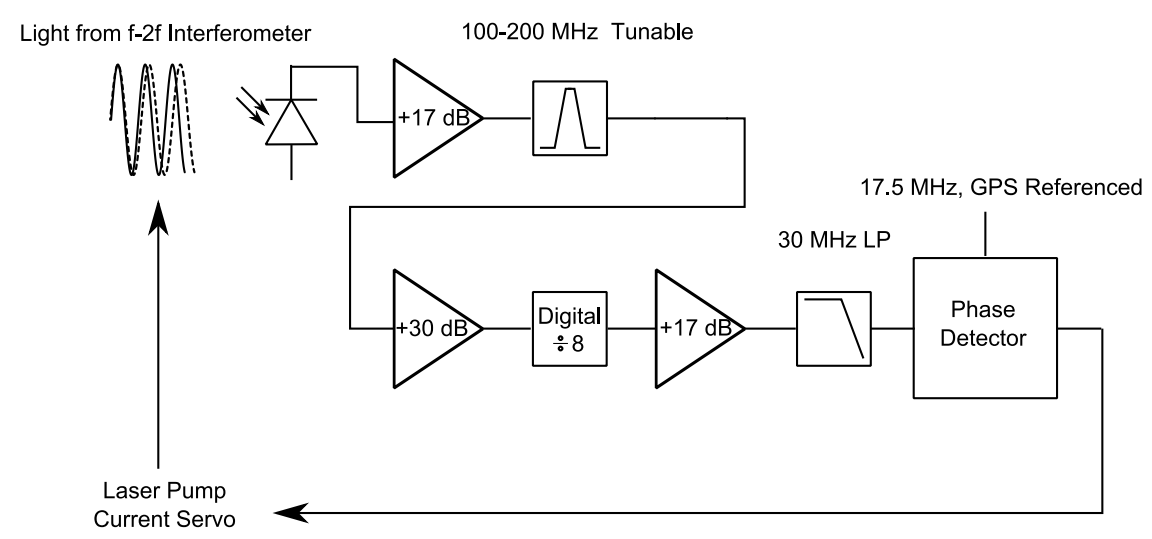

(b)

Figure 2: Locking electronics for stabilization of (a) $f_{\text {rep }}$ and (b) $f_{\text {ceo }}$, as described in the text. Feedback to $f_{\text {rep }}$ is applied by means of a piezo-mounted mirror inside the $250 \mathrm{MHz}$ laser cavity, while $f_{\text {ceo }}$ is locked by varying the current to the laser's pump diodes. 


\section{GENERATION OF A CALIBRATION FREQUENCY COMB}

A major challenge in developing a frequency comb for calibration of astronomical spectrographs is generating a mode spacing large enough to be well resolved by the spectrograph. For a spectrograph with resolving power $\lambda / \Delta \lambda=50,000$ at $1550 \mathrm{~nm}$, one resolution element, spanning 2 to 3 pixels on the detector, corresponds to roughly $3 \mathrm{GHz}$. An optimal calibration spectrum has one calibration line per 3 to 4 resolution elements, ${ }^{13}$ requiring a 12 to $16 \mathrm{GHz}$ mode spacing. While it is possible to build mode-locked lasers with repetition rates of up to $10 \mathrm{GHz},{ }^{14}$ it is difficult and such a laser requires precise alignment. An alternative route to obtaining a high mode spacing is the filtering of a mode-locked laser of lower repetition rate. ${ }^{8,15-18}$ This approach makes use of the resonances of Fabry-Pérot cavities, which have fundamental transverse modes at all frequencies where the accumulated round-trip phase is an integer multiple of $2 \pi \cdot{ }^{19}$ Neglecting dispersion in air and phase shifts occurring in the dielectric mirrors, the cavity resonance frequencies are

$$
f_{n}=n \frac{2 \pi c}{2 n_{\text {air }} L}
$$

where $L$ is the spacing between the mirrors comprising the cavity and $n_{\text {air }}$ is the index of refraction of air. The frequency $\pi c / n_{\text {air }} L$ can be set to a multiple $m$ of the the repetition rate of the mode-locked laser by tuning the cavity length, allowing only every $m^{\text {th }}$ comb mode to be transmitted. This increases the mode spacing to $m \times f_{\text {rep. }}$.

To reach a comb line spacing of $12.5 \mathrm{GHz}$, the spectrum is filtered by a factor of $m=50$. Fabry-Pérot cavities with a finesse of 2000 are used, with two dielectric mirrors with radii of curvature of $50 \mathrm{~cm}$ in a confocal configuration. ${ }^{19}$ To maintain transmission of every $50^{\text {th }}$ mode across $70 \mathrm{~nm}$ of bandwidth, the cavity lengths must be kept stable to better than $\pm 1 \mathrm{~nm}$. Cavity length control is achieved by mounting one mirror on a piezoelectric ring actuator, which provides a few micrometers of travel. For active locking, two methods were investigated: the Pound Drever Hall (PDH) technique ${ }^{20}$ and a dither-lock scheme. For the PDH lock, a fiber-coupled waveguide phase modulator was used to add small $17 \mathrm{MHz}$ offset sidebands to the comb. A circulator was used to direct the light reflected by the cavity onto a photodetector, which was then used to generate an error signal in the standard fashion. The dither technique is a lock-in scheme in which a small, $\sim 40 \mathrm{kHz}$ sinusoidal perturbation is imparted upon the cavity length. The intensity of the light transmitted by the cavity is photodetected, and a lock-in amplifier is used to create an error signal. The PDH technique has the advantage of imparting only small amplitude, RF modulation upon the comb, but at the expense of a loss of $\sim 50 \%$ of optical power coupling to the waveguide modulator and a distortion of the pulse shape caused by dispersion in the modulator. The dither-lock has the advantage of adding only a small loss caused by the perturbation of the cavity length, but imparts optical intensity noise at the dither frequency; see Fig 3. Ultimately, the loss and dispersion caused by the PDH lock were too great to outweigh its superior locking preformance, and the dither technique is used to lock both cavities.

After filtering the frequency comb, the spectrum spans only $70 \mathrm{~nm}$ centered around $1580 \mathrm{~nm}$; to cover the $\mathrm{H}$ band, the comb must be broadened. Broadening is accomplished in nonlinear optical fiber, ${ }^{21}$ where a large nonlinear coefficient promotes four wave-mixing, self phase modulation, and Raman shifts. ${ }^{22}$ In these parametric processes, three light fields interact to produce a fourth. To provide the necessary high peak optical power, the filtered comb light is amplified from $80 \mu \mathrm{W}$ to $1.4 \mathrm{~W}$. This amplification adds broad bandwidth optical intensity noise, arising from amplified spontaneous emission (ASE) in the gain medium. The presence of optical noise in the four wave mixing process causes optical fields from the comb to mix with noise fields, leading to a loss of coherence in the comb, as in Fig. 5(d). To remove the ASE, the amplified comb light is passed through a second filter cavity identical to the first, which rejects more than $99.9 \%$ of optical noise. To compensate for pulse broadening in single-mode fiber, a piece of dispersion compensating fiber (DCF) is inserted before the amplifiers. After optimizing fiber lengths, the system generates a $12.5 \mathrm{GHz}$ train of $400 \mathrm{fs}$ pulses (Fig 4) with an energy per pulse of $40 \mathrm{pJ}$. The pulses are sent through $50 \mathrm{~m}$ of highly nonlinear fiber, where it is broadened to span from 1350 to $1850 \mathrm{~nm}$. 


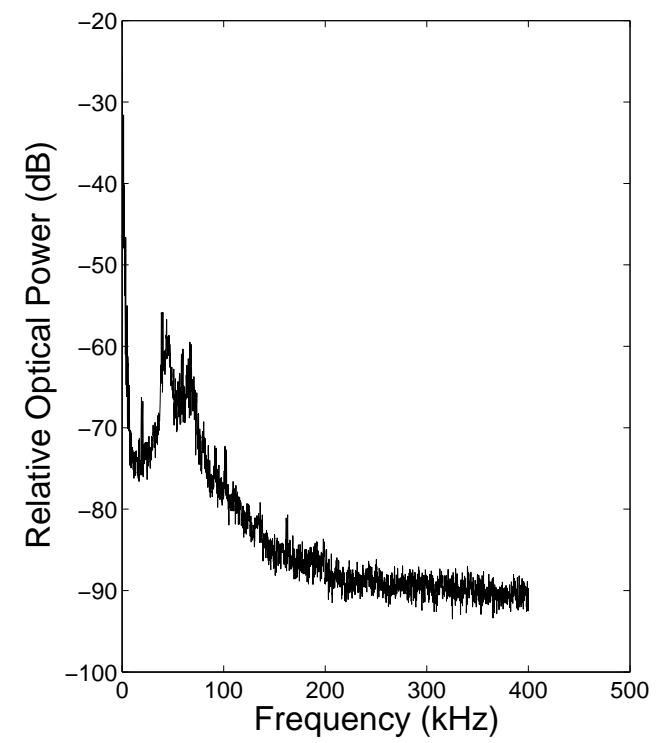

(a)

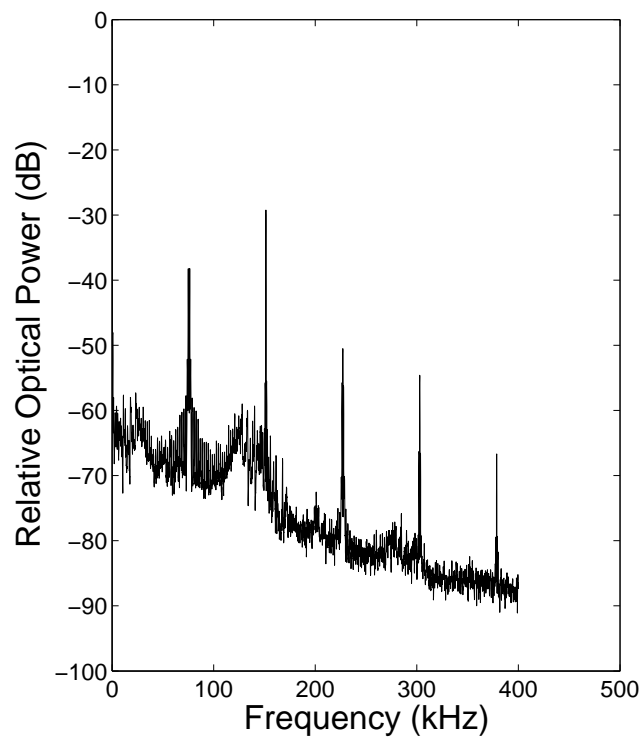

(b)

Figure 3: Optical intensity noise after filter cavity for(a) Pound-Driver-Hall and (b) cavity dither locking techniques. Peaks in the dither lock spectrum are harmonics of the dither frequency used to generate the error signal for locking.

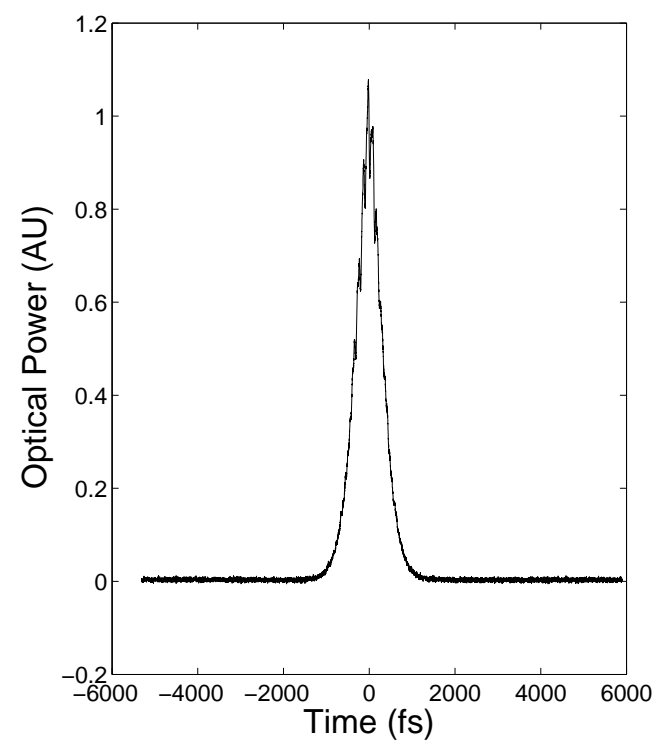

Figure 4: Autocorrelation of pulse entering highly nonlinear fiber. Pulse autocorrelation time is near the transform limit of $600 \mathrm{fs}$. 


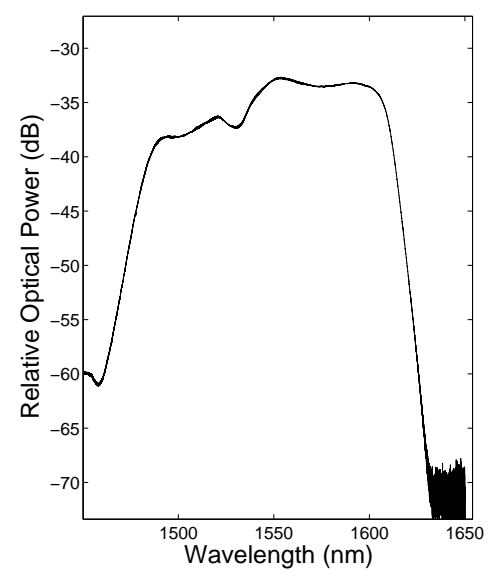

(a)

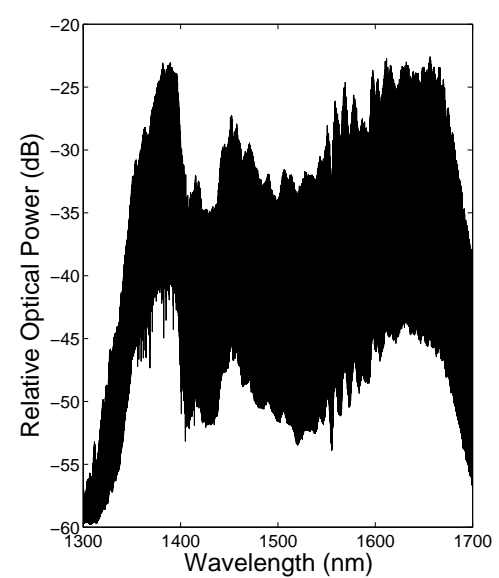

(c)

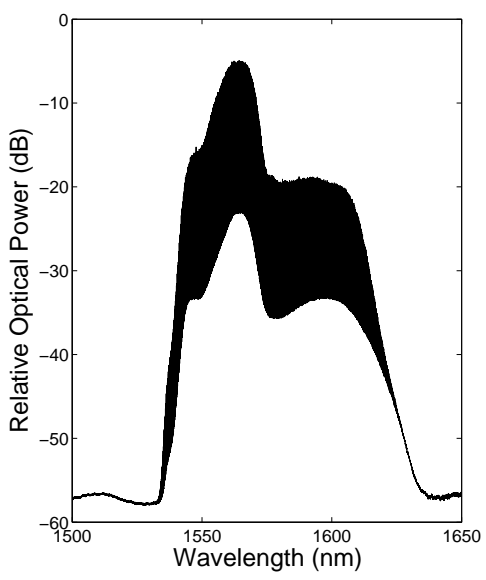

(b)

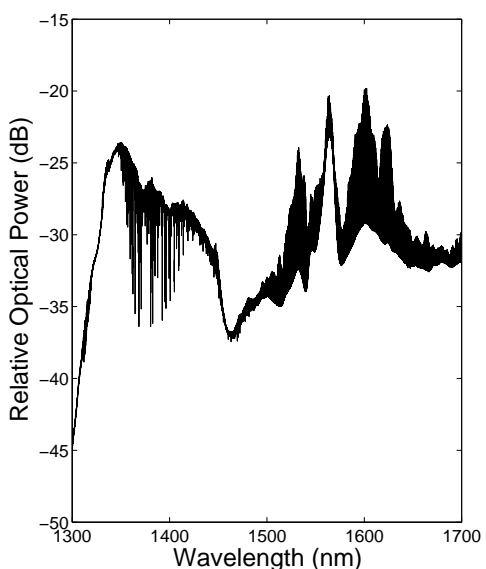

(d)

Figure 5: Optical spectra of the frequency comb through the optical system. The $250 \mathrm{MHz}$ frequency comb (a) is unresolved with our $\mathrm{R}=77,000$ optical spectrum analyzer; optical power is $34 \mathrm{~mW}$. After filtering and amplification, the modes are resolvable (b), and the total optical power is $1350 \mathrm{~mW}$. Filtering with a second cavity and nonlinear broadening (c) greatly increases the span of the spectrum, which goes beyond the range of the spectrum analyzer and has a total power of $390 \mathrm{~mW}$. Broadening the spectrum without the second filter cavity results in (d). Without the second filter cavity, mixing of optical noise with the frequency comb during broadening parametrically creates optical noise between comb modes, almost completely erasing the frequency comb. 


\section{MEASUREMENTS OF COMB}

Obtaining a calibration spectrum with $\mathrm{cm} / \mathrm{s}$ accuracy is challenging, even with a frequency comb. Filtering the frequency comb from $250 \mathrm{MHz}$ to $12.5 \mathrm{GHz}$ provides a comb with a spacing precisely 50 times larger, but also introduces ambiguity in the absolute frequency of $n \times f_{\text {rep }}$ in the remaining comb lines, where $n$ is a number between 1 and 50. To determine $n$, the frequency of a single $12.5 \mathrm{GHz}$ comb mode must be determined with an uncertainty of less than half of $f_{\mathrm{rep}}$, or $125 \mathrm{MHz}$. Such a measurement is performed nu use of a commercial wavemeter, with an accuracy better than $20 \mathrm{MHz}$ and an inexpensive fiber coupled distributed feedback (DFB) laser. The laser is tuned to the proximity of a comb line by adjusting the laser pump current while observing the optical heterodyne beat between the $\mathrm{CW}$ and laser comb on a photodiode. Simultaneously, the optical frequency of the CW laser is measured using the wavemeter. Sweeping the CW laser frequency either up or down while observing the beat frequency determines whether the CW laser has a higher or lower frequency than the nearest comb line. By using the CW laser frequency, the difference frequency between the $\mathrm{CW}$ laser and the comb, and the sign of the difference frequency, the absolute frequency of the comb mode can be determined to the accuracy of the wavemeter, and $n$ can be determined.

A more serious challenge is the characterization of the frequency comb after nonlinear broadening. The fourwave mixing process allows a mechanism for revival of the $250 \mathrm{MHz}$ comb modes nominally suppressed by the filter cavities. Because shifting of the apparent centers of the comb lines by only $10 \mathrm{kHz}$ affects the calibration at the $\mathrm{cm} / \mathrm{s}$ level, an accurate measurement of the side modes after broadening is critical to confidence in the comb. The largest side modes are the nearest neighbors $250 \mathrm{MHz}$ to either side of the $12.5 \mathrm{GHz}$ calibration lines, the magnitude and asymmetry of which can be directly determined by optical heterodyne. A narrow portion of the broadened comb spectrum is selected with a band-pass filter and combined with a reference beam from a tunable $\mathrm{CW}$ laser, then photodetected and electronically amplified. An RF spectrum analyzer records the spectrum, which is comprised of both the difference frequency signal between the tunable laser and a single comb mode and harmonics of $250 \mathrm{MHz}$ from the frequency comb alone. By examining the amplitudes of the side modes, as in Fig. 6, side-mode suppression and asymmetry can be measured. A careful series of measurements of the comb ${ }^{23}$ at $10 \mathrm{~nm}$ intervals across the spectrum from 1350 to $1700 \mathrm{~nm}$ indicates that across the broadened spectrum side mode asymmetry is less than $1 \mathrm{~dB}$, or $10 \%$.

Measuring the spectrum of the entire frequency comb with high resolution is a non-trivial task. Commercial optical spectrum analyzers with resolving power $\lambda / \Delta \lambda=77,000$ are capable of resolving the $12.5 \mathrm{GHz}$ comb modes, but are unable to determine line centers to better than a few GHz. A more complete measurement of the spectrum is desirable, as the nonlinear broadening process is complex, and it has been suggested ${ }^{24}$ that side modes could grow to the point where they dominate the nominally transmitted $12.5 \mathrm{GHz}$ comb. Accordingly, a measurement of the spectrum was made with the NIST 2 meter Fourier transform spectrometer. ${ }^{25}$ The spectrometer has a resolution of $70 \mathrm{MHz}$, and allows for the determination of the comb modes to better than 125 MHz. A preliminary analysis indicates that the spectrum maintains an even $250 \mathrm{MHz}$ spacing and demonstates that side modes are suppressed by more than a factor of $>20$ above $1700 \mathrm{~nm}$. Initial results are shown in Fig. 7 .

\section{CONCLUSION}

Laser frequency combs provide an ideal calibration source for high precision astronomical spectrographs. For this purpose, we demonstrate a frequency comb covering the $\mathrm{H}$ band, traceable to absolute frequency standards with a mode spacing of $12.5 \mathrm{GHz}$ or $0.42 \mathrm{~cm}^{-1}$. Measurements across the spectrum with optical heterodyne and Fourier transform spectroscopy demonstrate adequate suppresion of side modes. The comb frequency uncertainty is bounded by $\pm 30 \mathrm{kHz}$ (corresponding to a radial velocity of $5 \mathrm{~cm} / \mathrm{s}$ ), limited by the global positioning system disciplined oscillator reference. These results indicate that this comb can readily support radial velocity measurements below 1 meter per second in the near IR. 


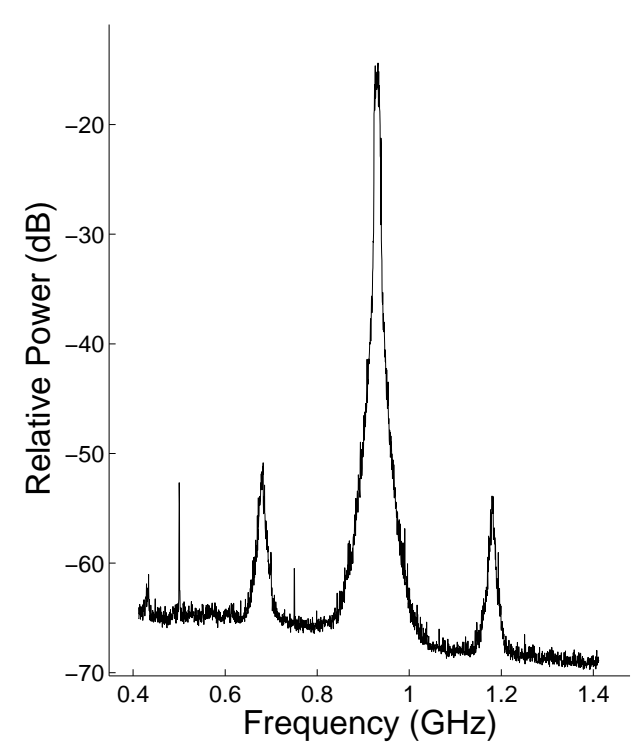

(a)

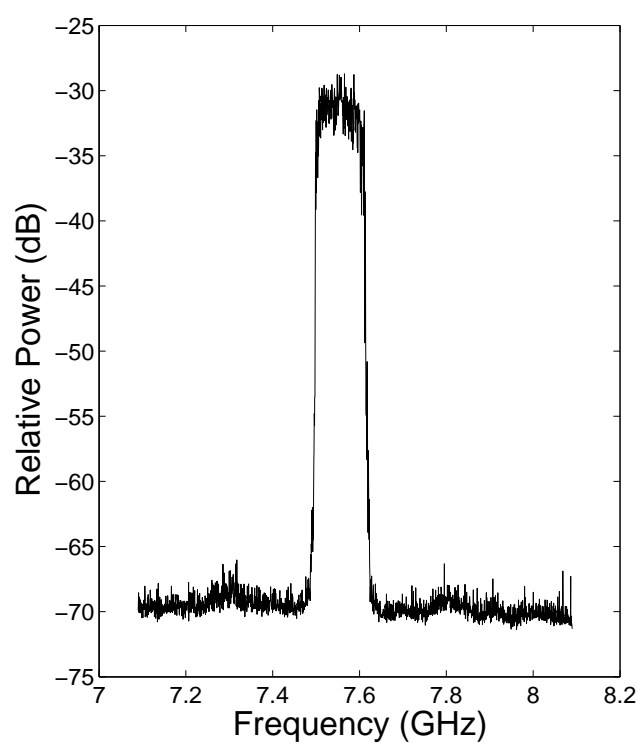

(b)

Figure 6: Heterodyne beat signals of individual frequency comb modes after nonlinear broadening at $1400.00 \mathrm{~nm}$ (a) and $1628.937 \mathrm{~nm}$ (b) with a tunable CW laser, measured with an RF spectrum analyzer. The central peak is the beat of the $\mathrm{CW}$ laser with the $12.5 \mathrm{GHz}$ filtered comb, the adjacent peaks are residual modes offset by $250 \mathrm{MHz}$. The visible asymmetry in (a) is due primarily to frequency dependent gain in the RF amplifier. By averaging measurements made with the tunable laser on either side of the comb line an asymmetry of less than $0.5 \mathrm{~dB}$ is measured, shifting the effective line center by less than $6 \mathrm{~cm} / \mathrm{s}$. The broad shape of the beat spectra are due to the relatively linewidth of the tunable lasers.

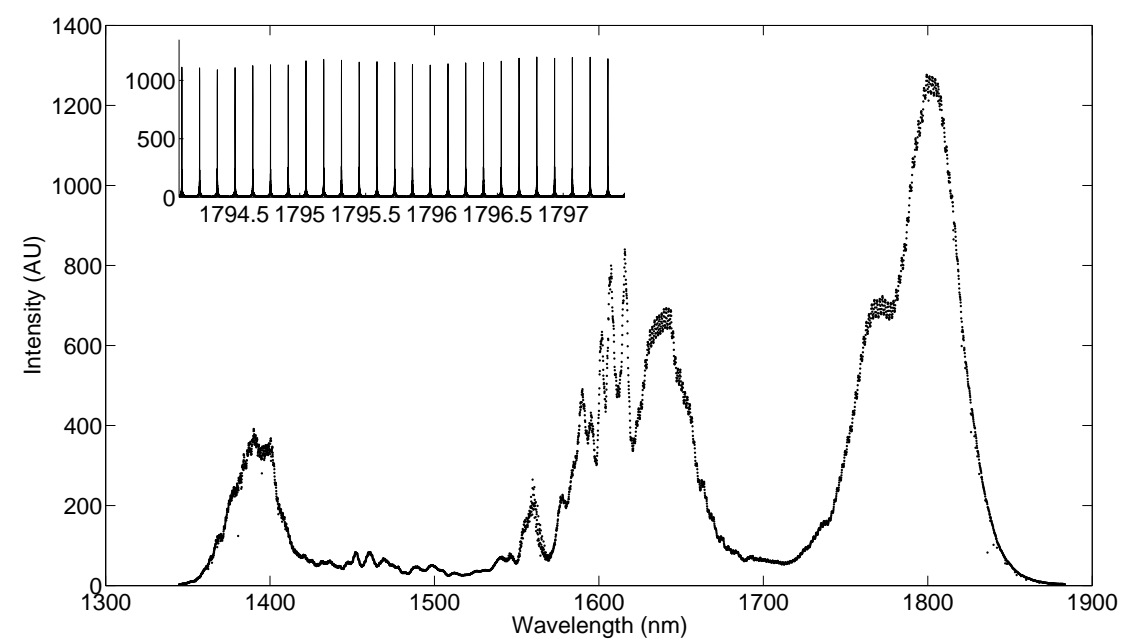

Figure 7: Preliminary analysis of measurement of the frequency comb with the NIST 2 meter Fourier transform spectrometer. The intensities of comb modes are plotted; approximately 5000 of them fill the spectrum. Inset: small section of the Fourier transform spectrogram. Small features around the base of each comb line are due to the instrument response of the FTS. 


\section{ACKNOWLEDGMENTS}

We acknowledge Craig J. Sansonetti for his assistance with the Fourier transform spectrometer measurements, Y.

Jiang and D. Braje for contributions in building the $250 \mathrm{MHz}$ comb source and M. Hirano of Sumitomo Electric Industries for use of the HNLF. Financial support is provided by NSF under Grant No. 0906034 and NIST. F. Quinlan is supported as an NRC/NAS postdoctoral fellow.

\section{REFERENCES}

[1] Mayor, M. and Queloz, D., "A Jupiter-mass companion to a solar-type star," Nature 378, 355-359 (1995).

[2] Eggenberger, A. and Udry, S., "Detection and Characterization of Extrasolar Planets through Doppler Spectroscopy," EAS Publications Series 41, 27-75 (2010).

[3] Udry, S., Fischer, D., and Queloz, D., "A Decade of Radial-Velocity Discoveries in the Exoplanet Domain," in [Protostars and Planets V], Reipurth, B., Jewitt, D., and Keil, K., eds., 685-699, University of Arizona Press (2007).

[4] Kasting, J. F., Whitmire, D. P., and Reynolds, R. T., "Habitable Zones around Main Sequence Stars," Icarus 101, 108-128 (1993).

[5] Reiners, A., Bean, J. L., Huber, K. F., Dreizler, S., Seifahrt, A., and Czesla, S., "Detecting Planets Around Very Low Mass Stars with the Radial Velocity Method," Astrophys. J 710(1), 432 (2010).

[6] Mayor, M. and Udry, S., "The quest for very low-mass planets," Phys. Scr. 2008(T130), 014010 (2008).

[7] Wright, J. T., Marcy, G. W., Fischer, D. A., Butler, R. P., Vogt, S. S., Tinney, C. G., Jones, H. R. A., Carter, B. D., Johnson, J. A., McCarthy, C., and Apps, K., "Four New Exoplanets and Hints of Additional Substellar Companions to Exoplanet Host Stars," Astrophys. J 657(1), 533 (2007).

[8] Braje, D. A., Kirchner, M. S., Osterman, S., Fortier, T., and Diddams, S. A., "Astronomical spectrograph calibration with broad-spectrum frequency combs," Eur. Phys. J. D 48, 57-66 (2008).

[9] Wilken, T., Lovis, C., Manescau, A., Steinmetz, T., Pasquini, L., Lo Curto, G., Hänsch, T. W., Holzwarth, R., and Udem, T., "High-precision calibration of spectrographs," Mon. Not. R. Astron. Soc. 405, L16-L20 (2010).

[10] Li, C.-H., Benedick, A. J., Fendel, P., Glenday, A. G., Kartner, F. X., Phillips, D. F., Sasselov, D., Szentgyorgyi, A., and Walsworth, R. L., "A laser frequency comb that enables radial velocity measurements with a precision of $1 \mathrm{~cm} \mathrm{~s}^{-1}, "$ Nature 452, 610-612 (2008).

[11] Wilken, T., Hnsch, T., Holzwarth, R., and P. Adel, M. M., "Low Phase Noise 250 MHz Repetition Rate Fiber fs Laser for Frequency Comb Applications," in [Proceedings of the CLEO/QELS Conference], (2007).

[12] Jones, D. J., Diddams, S. A., Ranka, J. K., Stentz, A., Windeler, R. S., Hall, J. L., and Cundiff, S. T., "Carrier-Envelope Phase Control of Femtosecond Mode-Locked Lasers and Direct Optical Frequency Synthesis," Science 288(5466), 635-639 (2000).

[13] Murphy, M. T., Udem, T., Holzwarth, R., Sizmann, A., Pasquini, L., Araujo-Hauck, C., Dekker, H., D'Odorico, S., Fischer, M., Hänsch, T. W., and Manescau, A., "High-precision wavelength calibration of astronomical spectrographs with laser frequency combs," Mon. Not. R. Astron. Soc. 380, 839-847 (2007).

[14] Bartels, A., Heinecke, D., and Diddams, S. A., "Passively mode-locked $10 \mathrm{GHz}$ femtosecond Ti:sapphire laser," Opt. Lett. 33(16), 1905-1907 (2008).

[15] Chen, J., Sickler, J. W., Fendel, P., Ippen, E. P., Kärtner, F. X., Wilken, T., Holzwarth, R., and Hänsch, T. W., "Generation of low-timing-jitter femtosecond pulse trains with $2 \mathrm{GHz}$ repetition rate via external repetition rate multiplication," Opt. Lett. 33, 959-+ (2008).

[16] Steinmetz, T., Wilken, T., Araujo-Hauck, C., Holzwarth, R., Hänsch, T., and Udem, T., "FabryPérot filter cavities for wide-spaced frequency combs with large spectral bandwidth," Appl. Phys. B: Lasers Opt. 96, $251-256$ (2009).

[17] Kirchner, M. S., Braje, D. A., Fortier, T. M., Weiner, A. M., Hollberg, L., and Diddams, S. A., "Generation of $20 \mathrm{GHz}$, sub-40 fs pulses at $960 \mathrm{~nm}$ via repetition-rate multiplication," Opt. Lett. 34, 872-+ (2009). 
[18] Sizer, II, T., "Increase in laser repetition rate by spectral selection," IEEE J. Quantum. Electron. 25, 97-103 (1989).

[19] Siegman, A., [Lasers], Univerity Science Books, Sausalito, CA (1986).

[20] Drever, R. W. P., Hall, J. L., Kowalski, F. V., Hough, J., Ford, G. M., Munley, A. J., and Ward, H., "Laser phase and frequency stabilization using an optical resonator," Applied Physics B: Lasers and Optics 31, 97-105 (1983).

[21] Hirano, M., Nakanishi, T., Okuno, T., and Onishi, M., "Silica-Based Highly Nonlinear Fibers and Their Application," IEEE J. Sel. Top. Quantum Electron. 15, 103 -113 (2009).

[22] Agrawal, G., [Nonlinear Fiber Optics], Academic Press, Burlington, MA (2007).

[23] Quinlan, F., Ycas, G., Osterman, S., and Diddams, S. A., "A 12.5 GHz-spaced optical frequency comb spanning > $400 \mathrm{~nm}$ for near-infrared astronomical spectrograph calibration," Rev. Sci. Instrum. 81(6), 063105 (2010).

[24] Chang, G., Li, C.-H., Phillips, D. F., Walsworth, R. L., and Kärtner, F. X., "Toward a broadband astrocomb: effects of nonlinear spectral broadening in optical fibers," Opt. Express 18(12), 12736-12747 (2010).

[25] Nave, G., Sansonetti, C. J., and Griesmann, U., "Progress on the NIST IR-vis-UV Fourier transform spectrometer," in [Fourier Transform Spectroscopy: Methods and Applications], OSA Technical Digest Series 3, 38-40, Optical Society of America, Washington, D.C. (1997). 